\title{
A New Way of Understanding why the Harmonic Series is Divergent
}

\author{
Salman Mahmud* \\ Student of Government Azizul Haque College, Bogura, Bangladesh
}

*Corresponding Author: Salman Mahmud, Student of Government Azizul Haque College, Bogura, Bangladesh

\begin{abstract}
The harmonic series is one of the most important series in mathematics. Here in this short paper we will establish a new lemma to prove that the harmonic series is divergent. The main purpose of establishing this lemma is to demonstrate a new way of understanding why the harmonic series must be divergent.
\end{abstract}

\section{INTRODUCTION}

We know the harmonic series is divergent. There are several well-known proofs of the divergence of the harmonic series. But here we will show a different kind of proof which will help us to easily understand why the harmonic series is divergent. Here we will establish a new lemma which will be helpful to understand for some infinite series like harmonic series that the series is divergent or not.

\section{Proposing the Lemma}

Lemma 1: Suppose a series $S_{1}=a_{1}+a_{2}+a_{3}+\cdots$ and another convergent series $S_{2}=b_{1}+b_{2}+$ $b_{3}+\cdots$ where $S_{2} \neq 0$. After adding the series $S_{2}$ with the series $S_{1}$ or subtracting the series $S_{2}$ from the series $S_{1}$ if all the nonzero terms of $S_{1}+S_{2}$ or $S_{1}-S_{2}$ become the same as the nonzero terms of $S_{1}$, then $S_{1}$ must be a divergent series.

Proof: If all the nonzero terms of $S_{1}+S_{2}$ or $S_{1}-S_{2}$ become the same as the nonzero terms of $S_{1}$, then it will be true if and only if $S_{1}=a_{1}+a_{2}+a_{3}+\cdots=+\infty$ or $S_{1}=a_{1}+a_{2}+a_{3}+\cdots=$ $-\infty$ because if we add anything or subtract anything from infinity $(+\infty$ or $-\infty)$, the result will be infinity $(+\infty$ or $-\infty)$. If $S_{1}$ is a convergent series then the nonzero terms of $S_{1}+S_{2}$ or $S_{1}-S_{2}$ will not become the same as the nonzero terms of $S_{1}$.

This lemma is not helpful for every divergent series. It would be applicable for some infinite series to check that the series is divergent or convergent. Now, let us apply this lemma for harmonic series.

$$
\begin{gathered}
S_{1}=1+\frac{1}{2}+\frac{1}{3}+\frac{1}{4}+\frac{1}{5}+\frac{1}{6}+\cdots \\
S_{2}=-\left(1-\frac{1}{2}+\frac{1}{3}-\frac{1}{4}+\frac{1}{5}-\frac{1}{6}+\cdots\right)=-\ln 2 \\
1+\frac{1}{2}+\frac{1}{3}+\cdots
\end{gathered}
$$

Here, after subtracting the series $1-\frac{1}{2}+\frac{1}{3}-\frac{1}{4}+\frac{1}{5}-\frac{1}{6}+\cdots$ from the harmonic series, the nonzero terms of $S_{1}-S_{2}$ become the same as the nonzero terms of the harmonic series. So, the harmonic series is divergent.

\section{CONCLUSION}

The proof of the divergence of the harmonic series showed in the paper will help us to understand why the Harmonic series is divergent from a new perspective. 


\section{ACKNOWLEDGEMENT}

I should Thanks MD. Shah Alam and MST. Sabina Yesmin for providing me with the help materials.

\section{REFERENCES}

[1] Wikipedia n. d., Harmonic Series (mathematics), viewed 6 December 2020, https://en.wikipedia.org/ wiki/Harmonic_series_(mathematics)

\section{AUTHOR'S BIOGRAPHY}

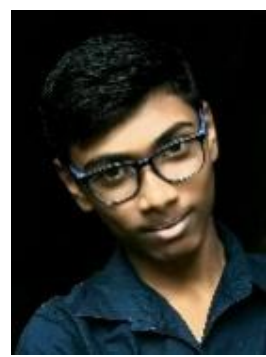

This is Salman Mahmud. I am an undergraduate student of Government Azizul Haque College, Bogura, Bangladesh (2020). My core research interest is in modern physics and pure mathematics. But I am interested in all the sectors of science. I have contributed in so many researches. I like to spend time with my parents MD. Shah Alam and MST Sabina Yesmin. In my leisure period I like to play video games.

Citation: Salman Mahmud, A New Way of Understanding why the Harmonic Series is Divergent, International Journal of Scientific and Innovative Mathematical Research (IJSIMR), vol. 8, no. 10, pp. 23-24, 2020. Available : DOI: https://doi.org/ 10.20431/2347-3142.0810004

Copyright: (C) 2020 Authors. This is an open-access article distributed under the terms of the Creative Commons Attribution License, which permits unrestricted use, distribution, and reproduction in any medium, provided the original author and source are credited. 